\title{
A Nonlinear Alternating Direction Method
}

\author{
By R. B. Kellogg*
}

Abstract. An alternating direction iteration method is formulated, and convergence is proved, for the solution of certain systems of nonlinear equations. The method is applied to a heat conduction problem with a nonlinear boundary condition.

1. Alternating direction methods are often used for solving the sets of linear equations arising from the discretization of elliptic boundary value problems [1], [2], [3]. In this paper, an alternating direction method is formulated for a certain nonlinear system of equations. Convergence of the method is established in the case of a single iteration parameter. Finally, the method is applied to a set of equations arising from a steady-state heat conduction problem with nonlinear boundary conditions. Such boundary conditions occur when energy is transmitted from the boundary of the region by means of radiation or by means of natural convection to a liquid $[4$, p. 21].

2. It is desired to solve the system of $n$ equations in $n$ unknowns

$$
A(u)=0,
$$

where $A$ is a continuous, not necessarily linear, vector-valued function of the real $n$-dimensional vector $u$. Letting $H(u)$ and $V(u)$ be continuous vector functions such that $A(u)=H(u)+V(u)$, the iterative scheme under consideration is

$$
\begin{aligned}
r u^{n+1 / 2}+H\left(u^{n+1 / 2}\right) & =r u^{n}-V\left(u^{n}\right) \\
r u^{n+1}+V\left(u^{n+1}\right) & =r u^{n+1 / 2}-H\left(u^{n+1 / 2}\right), \quad n=0,1, \cdots,
\end{aligned}
$$

where $u^{0}$ is an arbitrary guess of the solution of (1), and where $r>0$ is fixed throughout the rest of this paper. It will be shown that under certain conditions, (1) has a unique solution $u$, (2) may be solved uniquely for $u^{n+1 / 2}$ and $u^{n+1}$, and $u^{n}$ converges to $u$.

Let $(u, v)$ and $\|u\|$ denote respectively the usual inner product and norm of the real vectors $u$ and $v$. A function $B$ is called strongly monotone if there is a $c>0$ such that

$$
(B(u)-B(v), u-v) \geqq c\|u-v\|^{2}, \quad \text { all } u, v .
$$

If $c=0$ in (3), $B$ is called monotone. (In a complex space, (3) is modified by taking the real part of the inner product.) The analysis of (2) relies on the following theorem of Minty [5].

THEOREM 1. If $B$ is continuous and strongly monotone, then $B u=f$ has a unique solution for any $f$.

Received June 5, 1968.

* This research was supported in part by Atomic Energy Commission Grant AEC-AT(40-1)3443, and National Science Foundation Grant NSF GP-3666. 
This result is true, when properly stated, in infinite-dimensional spaces. A proof in the finite-dimensional case may be found, for example, in [6, p. 69].

It will be assumed that $H$ and $V$ are monotone, and either $H$ or $V$ is strongly monotone. Then $A$ is strongly monotone and from Theorem 1, (1) has a unique solution. Also, letting $I$ be the identity operator, $I u=u$, the functions $r I+H$ and $r I+V$ are strongly monotone. This gives the following lemma.

Lemma 1. If $H$ and $V$ are continuous and monotone, (2) defines, for each $u^{0}, a$ sequence $u^{1 / 2}, u^{1}, \cdots$.

If $B$ is continuous and monotone, Theorem 1 allows us to define $(r I+B)^{-1}$ to be the inverse operator to $r I+B$. We set $T_{B}=(r I-B)(r I+B)^{-1} . B$ is said to satisfy a Lipschitz condition on a set $S$ if there is a constant $K>0$ such that $\|B(u)-B(v)\| \leqq K\|u-v\|$ when $u \in S, v \in S$. Then we have the following result.

Lemma 2. If $B$ is monotone, then $T_{B}$ satisfies a Lipschitz condition with constant 1 . If $B$ is strongly monotone and satisfies a Lipschitz condition on bounded sets, then on any bounded set, $T_{B}$ satisfies a Lipschitz condition with constant $<1$.

Proof. Let $v, w$ be given, and let $v_{1}=(r I+B)^{-1} v, w_{1}=(r I+B)^{-1} w$. Then

$$
\frac{\left\|T_{B}(v)-T_{B}(w)\right\|^{2}}{\|v-w\|^{2}}=\frac{\left\|r v_{1}-B\left(v_{1}\right)-r w_{1}+B\left(w_{1}\right)\right\|^{2}}{\left\|r v_{1}+B\left(v_{1}\right)-r w_{1}-B\left(w_{1}\right)\right\|^{2}}=\frac{a-b}{a+b} \leqq 1,
$$

where

$$
a=r^{2}+\frac{\left\|B\left(v_{1}\right)-B\left(w_{1}\right)\right\|^{2}}{\left\|v_{1}-w_{1}\right\|^{2}}, \quad b=2 r \frac{\left(B\left(v_{1}\right)-B\left(w_{1}\right), v_{1}-w_{1}\right)}{\left\|v_{1}-w_{1}\right\|^{2}} .
$$

Hence $T_{B}$ satisfies a Lipschitz condition with constant 1 . It is easily seen that $a \geqq b \geqq 2 r c$, where $c$ is the constant of (3). If $v$ and $w$ are restricted to a bounded set, then $v_{1}$ and $w_{1}$ lie in a bounded set of vectors. Suppose that, in this set, $B$ satisfies a Lipschitz condition with constant $K$. Then $a \leqq r^{2}+K^{2}$, and

$$
\frac{\left\|T_{B}(v)-T_{B}(w)\right\|^{2}}{\|v-w\|^{2}}=\frac{a-b}{a+b} \leqq \frac{r^{2}+K^{2}-2 r c}{r^{2}+K^{2}+2 r c}<1 .
$$

This completes the proof of the lemma.

The convergence theorem for the alternating direction scheme (2) can now be given.

Theorem 2. If $H$ and $V$ are monotone and either $H$ or $V$ is strongly monotone and satisfies a Lipschitz condition on bounded sets, then for any $u^{0}$, the sequence $u^{1 / 2}, u^{1}, \ldots$ defined by (2) converges to the unique solution $u$ of (1).

Proof. Defining $v^{n}=(r I+V)\left(u^{n}\right), v^{n+1 / 2}=(r I+H)\left(u^{n+1 / 2}\right)$, (2) may be written

$$
v^{n+1 / 2}=T_{V}\left(v^{n}\right), \quad v^{n+1}=T_{H}\left(v^{n+1 / 2}\right) .
$$

Similarly, defining $v=(r I+V)(u), w=(r I+H)$, we have from (1)

$$
w=T_{V}(v), \quad v=T_{I I}(w) .
$$

From (4), (5), and Lemma $2,\left\|v^{n}-v\right\| \leqq\left\|v^{1 / 2}-w\right\|,\left\|v^{n+1 / 2}-w\right\| \leqq\left\|v^{1 / 2}-w\right\|$, for $n=1,2, \cdots$, so the vectors $v, w$, and $v^{j}, j=0, \frac{1}{2}, 1, \cdots$, all lie in a bounded set. Appealing again to (4), (5), and Lemma 2, we find that there is a positive constant $p<1$ such that $\left\|v^{n+1}-v\right\| \leqq p\left\|v^{n}-v\right\|$. It follows that $v^{n} \rightarrow v, v^{n+1 / 2} \rightarrow w$. Since 
$(r I+H)^{-1}$ and $(r I+V)^{-1}$ are continuous, $u^{j} \rightarrow u$ as $j \rightarrow \infty$ through integer and half integer values.

The proof of this theorem actually shows that the convergence is geometric, $\left\|u^{j}-u\right\|=O\left(p^{j}\right)$. To implement these iterations one must solve the nonlinear equations (2). It will be shown how this is possible in certain cases.

3. To apply the iterative method (2) we consider in a domain $D$ of the $x y$ plane the boundary value problem

$$
\begin{aligned}
-\nabla(k(x, y) \nabla T(x, y)) & =q(x, y), \quad \text { in } D \\
-k(x, y) \partial T(x, y) / \partial n & =g(x, y, T(x, y)), \quad \text { on } \partial D
\end{aligned}
$$

where $k>0$ in $D$, and $n$ is the outward pointing unit normal vector to the boundary $\partial D$ of $D$. Such a problem may arise, for example, in the theory of nonlinear heat conduction [4, p. 21]. We will assume that for each $(x, y) \in \partial D$ and for each $M>0$, $g(x, y, T)$ satisfies a Lipschitz condition in $T$ when $|T|<M$. Also, we assume that for some $d>0$ and any real $T_{1}$ and $T_{2}$,

$$
\frac{g\left(x, y, T_{2}\right)-g\left(x, y, T_{1}\right)}{T_{2}-T_{1}} \geqq d, \quad(x, y) \in \partial D .
$$

To obtain a difference approximation to (6), (7), we assume that $\partial D$ is a polygon and that there is superimposed on the $x y$ plane a rectangular grid of mesh lines drawn parallel to the $x$ and $y$ axes and dividing the $x y$ plane into a set of rectangles such that if the polygon $D$ intersects one of the rectangles, the intersection must be either a vertex, a side, or a diagonal of the rectangle. We employ a commonly used difference approximation, discussed for example in [3], but modified to take into account the nonlinear boundary condition (7). In this difference approximation the unknowns are the values $u(P)$ of the approximate temperature $u$ at the mesh points $P \in \bar{D}$. To describe the difference approximation we let $A_{0}$ be the matrix of coefficients of the difference approximation [3] of the linear boundary value problem consisting of (6) with the boundary condition $\partial T / \partial n=0$. Then discretizing the nonlinear problem (6), (7), gives rise to the nonlinear system of equations $A(u)=0$ where $A(u)=A_{0} u-\bar{q}+C(u)$, with $\bar{q}$ a constant vector and $C(u)$ defined, at any mesh point $P \in \bar{D}$, by

$$
\begin{aligned}
C(u)(P) & =0, \quad P \notin \partial D \\
& =l(P) g(x, y, u(P)), \quad P=(x, y) \in \partial D .
\end{aligned}
$$

The positive quantity $l(P)$ is defined for each mesh point $P \in \partial D$ by

$$
l(P)=\frac{1}{2}\left(\overline{P P}_{1}+\overline{P P}_{2}\right)
$$

where $P_{1}$ and $P_{2}$ are the two mesh points on $\partial D$ lying next to and on either side of $P$.

Following [3] we write $A_{0}=H_{0}+V_{0}$ where $H_{0}$ and $V_{0}$ are symmetric, positive semidefinite matrices. Similarly, we define for each mesh point $P \in \partial D, l(P)=$ $l_{I I}(P)+l_{V}(P)$ where, if $P, P_{1}$, and $P_{2}$ are as in (10), and if $\theta_{j}$ is the angle the line $P P_{j}$ makes with the $x$-axis, then

$$
\begin{aligned}
& l_{H}(P)=\left(\overline{P P}_{1} \cos ^{2} \theta_{1}+\overline{P P}_{2} \cos ^{2} \theta_{2}\right), \quad P \in \partial D \\
& l_{V}(P)=l(P)-l_{H}(P) .
\end{aligned}
$$


As in (9) we define $C_{H}(u)$ and $C_{V}(u)$ with $C=C_{H}+C_{V}$, and we define

$$
\begin{aligned}
& H(u)=H_{0} u+C_{H}(u)-\frac{1}{2} \bar{q}, \\
& V(u)=V_{0} u+C_{V}(u)-\frac{1}{2} \bar{q} .
\end{aligned}
$$

(See also [3, p. 223].) With these definitions we have the following lemma.

Lemma 3. $A, H$, and $V$ are continuous and strongly monotone.

Proof. Continuity follows from continuity of $g(x, y, T)$ as a function of $T$. To prove that $A$ is monotone, note that from (8),

$$
(A(u)-A(v), u-v) \geqq\left(A_{0}(u-v), u-v\right)+d \sum_{P \in \partial D}(u(P)-v(P))^{2} .
$$

The right side of (12) is a nonnegative quadratic form in $z=u-v$ which may be written $\left(A_{1} z, z\right)$. The matrix $A_{1}$ is easily found to be a nonsingular $M$ matrix [7]. Hence $A_{1}$ is positive definite and $A$ is strongly monotone. $H$ and $V$ are treated in the same way.

Using Lemma 3 and Theorem 2 we deduce immediately

Theorem 3. If $H$ and $V$ are given by (11) and $u^{0}$ is arbitrary, the iterations $u^{k}$, $k=\frac{1}{2}, 1, \cdots$, given by $(2)$ converge to the unique solution $u$ of $A(u)=0$.

4. For the iterative scheme to be of use we must be able to solve equations of the form

$$
\begin{array}{r}
r v+H(v)=\xi \\
r w+V(w)=\eta
\end{array}
$$

where $H, V$ are given by (11). This may be accomplished by the following trick, suggested by $\mathrm{W}$. Guilinger. The component of $r v+H(v)$ at a mesh point $\left(x_{j}, y_{i}\right)$ depends only on the values of $v$ at mesh points $\left(x_{k}, y_{i}\right)$ on the same horizontal line. Thus, to solve (13), it suffices to consider equations of the form

$$
r v_{i}+H_{0, i} v_{i}+C_{H, i}\left(v_{i}\right)=\xi_{i},
$$

the subscript $i$ denoting projection onto the $i$ th horizontal line. $r I+H_{0, i}$ is a tridiagonal matrix and hence easily invertible. Calling the inverse $E_{i}$, we write

$$
v_{i}=E_{i} \xi_{i}-E_{i} C_{H, i}\left(v_{i}\right) \text {. }
$$

Suppose the $i$ th horizontal line meets $D$ at only two mesh points, the points on columns $j_{0}$ and $j_{1}$. The vector $C_{H, i}\left(v_{i}\right)$ has components all 0 except at the points on columns $j_{0}$ and $j_{1}$. Hence one may extract from (16) a simultaneous pair of equations in the unknowns $v_{j_{0}, i}$ and $v_{j_{1}, i}$. When these are solved, the remaining components of $v_{i}$ may be found from (16). The solution of (15) has thus been reduced to the solution of two sets of tridiagonal linear equations, plus a set of simultaneous nonlinear equations in two scalar variables. Equation (14) is handled in the same way.

If the function $g(x, y, T)$ is continuously differentiable in $T$ and if $\partial g / \partial T>0$, then it may be shown that the nonlinear pair of equations extracted from (16) may be solved by Newton's method and convergence is assured with any initial guess. To see this, note that with the hypotheses on $g$, (15) may be solved for $v_{i}$ by Newton's method and convergence is guaranteed. (See, for example, [8] or [9].) The Newton iterations for (15) may be written 


$$
\left(r I+H_{0, i}+C_{H, i}^{\prime}\left(v_{i}^{n}\right)\right)\left(v_{i}^{n+1}-v_{i}^{n}\right)=\xi_{i}-r v_{i}^{n}-H_{0, i} v_{i}^{n}-C_{H, i}\left(v_{i}{ }^{n}\right),
$$

or

$$
v_{i}^{n+1}=E_{i} \xi_{i}-E_{i} C_{H, i}^{\prime}\left(v_{i}{ }^{n}\right)-E_{i} C_{H, i}\left(v_{i}{ }^{n}\right)\left(v_{i}{ }^{n+1}-v_{i}{ }^{n}\right) .
$$

Again, one may extract from (17) a pair of equations for the components of $v_{i}{ }^{n+1}$ at the mesh points $\left(j_{0}, i\right)$ and $\left(j_{1}, i\right)$. These equations are the Newton iteration equations of the nonlinear system extracted from (16).

University of Maryland

College Park, Maryland 20742

1. D. W. Peaceman \& H. H. Rachford, JR., "The numerical solution of parabolic and elliptic differential equations," $J$. Soc. Indust. Appl. Math., v. 3, 1955, pp. 28-41. MR 17, 196.

2. G. Birkhoff, R. S. VARGA \& D. Young, "Alternating direction implicit methods," $A d-$ vances in Computers, Vol. 3, Academic Press, New York, 1962, pp. 189-273. MR 29 \#5395.

3. J. Spanier, "Alternating direction methods applied to heat conduction problems," Mathematical Methods for Digital Computers, Vol. 2, Wiley, New York, 1967.

4. H. S. Carslaw \& J. C. JAEger, Conduction of Heat in Solids, Oxford Univ. Press, London, 1947. MR 9, 188.

5. G. J. MiNTY, "Monotone (nonlinear) operators in Hilbert space," Duke Math. J., v. 29, 1962, pp. 341-346. MR $29 \# 6319$.

6. D. G. DeFigueiredo, Topics in Nonlinear Functional Analysis, Institute for Fluid Dynamics \& Applied Mathematics, no. 48, Lecture Series, Univ. of Maryland, 1967.

7. R. S. VARGA, Matrix Iterative Analysis, Prentice-Hall, Englewood Cliffs, N. J., 1962. MR $28 \# 1725$.

8. D. Greenspan \& S. PARter, "Mildly nonlinear elliptic partial differential equations and their numerical solution. II," Numer. Math., v. 7, 1965, pp. 129-146. MR 31 \#1777.

9. J. M. Ortega \& W. C. RheinboldT, "Monotone iterations for nonlinear equations with application to Gauss-Seidel methods," SIAM J. Numer. Anal., v. 4, 1967, pp. 171-190. MR 35 \#6328. 